\title{
Correct Definition of Sound Speed and Its Consequences in the Tasks of Hydrodynamics
}

\author{
Vladimir Kirtskhalia \\ I. Vekua Sokhumi Institute of Physics and Technology (SIPT), 7 Mindeli St., 0186 Tbilisi, Georgia \\ Correspondence should be addressed to Vladimir Kirtskhalia; v.kirtskhalia@gmail.com
}

Received 20 June 2016; Accepted 18 September 2016

Academic Editor: Ciprian Iliescu

Copyright ( 2016 Vladimir Kirtskhalia. This is an open access article distributed under the Creative Commons Attribution License, which permits unrestricted use, distribution, and reproduction in any medium, provided the original work is properly cited.

\begin{abstract}
The paper considers the applied problems of hydrodynamics and based on the new results, published by the author in recent years, shows that main assumptions used in the course of their solution, namely, incompressibility of liquids and potentiality of their movement, are not applicable to liquids in the gravitational field of the Earth.
\end{abstract}

\section{Introduction}

Surface gravity waves are generated and propagated on the interface of two liquids; therefore, while studying them, the methods of theory of hydrodynamic tangential gap are applied. Such waves arise in consequence of disturbance of these surfaces and depending on the volume of disturbance, they may be either linear (when disturbances of parameters of liquids are much less than their equilibrium value) or nonlinear (when disturbances of parameters of liquids are bigger or of the order of their equilibrium value). A great number of scientific publications have been dedicated to the study of this problem, which stemmed from the second half of the 18th century. The vast majority of these works rely on two assumptions: (a) liquids are incompressible (i.e., their densities are constant) and (b) liquid motions are potential, that is, noncircuital.

The first assumption apparently contradicts the concept of mechanical wave in medium itself, since it is known that a wave is propagated only when, under disturbance of density of medium in any point, the elastic forces originate, spreading these perturbations to other points. If the medium is incompressible within the above sense, then any perturbation will be propagated with infinite velocity and thus, generation of wave is impossible. Irrelevance of condition of incompressibility to liquids was shown in work [1]. Upon correct determination of sound speed [2], according to which its true (real) value in the medium is determined by combination of adiabatic $\left(C_{s}\right)$ and isobaric $\left(C_{p}\right)$ sound speeds, it became evident that compressibility and incompressibility have thermodynamic rather than mechanic meaning and they are directly related to homogeneity or inhomogeneity of the medium [3]. Homogeneous medium, which is free from the influence of external force field, is always compressible and sound speed in it is adiabatic. Strongly inhomogeneous medium, which is largely influenced by external force filed, is always incompressible and sound speed in it is isobaric. These results led to necessity of changing the mass conservation equation. It appeared that the equation in its current form is fair only for homogeneous medium and as any medium in gravity field is more or less inhomogeneous, it needs to be generalized. The generalized mass conservation equation is obtained in work [4], in the right part of which an additional summand is entered determining the change of density of constant mass, under isobaric change of its volume as a result of temperature variations conditioned by entropy oscillations in inhomogeneous (nonisentropic) medium. When the first summand equals zero, which is the condition for incompressibility of the medium, the second summand remains, equaling isobaric change of its density. Disregard of this summand leads to existence of internal gravity wave [5] (\$13) whose nature is unclear [3].

Fallacy of the second assumption is proved by the Thompson theorem (1869), according to which liquid motion in the 
gravity field of the Earth cannot be potential, since it represents a nonisentropic medium [5] (\$9). Notwithstanding this, the author himself contradicts this theorem when solving the linear problem of capillary-gravity waves, where motions of liquids are potential [5] (\$62). Apparently, he opted for the Helmholtz theorem, according to which circulation of speed vector in gravity field is preserved [6] (\$1.2).

Discussion of this problem with particular intensity started from 2012, after publication of work [2]. The subsequent papers based on the critical analysis of the works of other authors persuasively proved existence of the aforementioned contradictions, suggesting the ways of overcoming them. Although those papers received highly favorable reviews, they were neglected by the scientific community. This is evidenced by the fact that they are scarcely cited. For example, we give several studies [7-18], published after 2012, and, as before, consider liquids as incompressible and their motion as potential. Work [12] is worthy of particular regret, as it represents lecture notes and is designed for students.

The aim of the proposed article is to once again present to the experts of this field of physics the overview of earlier published works, with some refinements and additions, to draw their attention to the problems of gas and hydrodynamics.

1.1. The Generalized Equation of Conservation of Mass and System of Hydrodynamic Equations in Gravity Field of the Earth. The principal equations describing liquid motion in gravity field of the Earth are the following:

(1) Euler's motion equation:

$$
\rho \frac{d \vec{V}}{d t}=\rho\left[\frac{\partial \vec{V}}{\partial t}+(\vec{V} \nabla) \vec{V}\right]=-\nabla P+\rho \vec{g} .
$$

(2) Mass conservation equation:

$$
\frac{d \rho}{d t}=\frac{\partial \rho}{\partial t}+(\vec{V} \nabla) \rho=-\rho \nabla \vec{V}
$$

(3) Adiabatic equation:

$$
\frac{\partial s}{\partial t}+(\vec{V} \nabla s)=0
$$

Equation (2) determines change of density of liquid particle in given, immovable volume and says that it equals difference of mass flows incoming and outgoing through surface restricting this volume. However, change of density may occur also at the expense of change of volume of constant mass of substance. Indeed,

$$
\begin{aligned}
\frac{d \rho}{d t} & =\frac{d}{d t}\left(\frac{m}{v}\right)=\frac{v(d m / d t)-m(d v / d t)}{v^{2}} \\
& =\left(\frac{d \rho}{d t}\right)_{v}-\left(\rho \frac{d}{d t} \ln v\right)_{m} .
\end{aligned}
$$

Assuming here $m=1$, that is, $v=1 / \rho$, we will get

$$
\left(\rho \frac{d}{d t} \ln v\right)_{m}=-\left(\rho \frac{d}{d t} \ln \rho\right)_{m}=-\left(\frac{d \rho}{d t}\right)_{m} .
$$

And finally, from (4), we will find

$$
\frac{d \rho}{d t}=\left(\frac{\partial \rho}{\partial t}\right)_{v}+\left(\frac{\partial \rho}{\partial t}\right)_{m} .
$$

Thus, complete change of density consists of two parts, first of which is determined by (2). The second part describes change of density of substance of constant mass as a consequence of isobaric change of volume which can occur only at the cost of change of temperature, which in turn, in absence of heat source, is possible only in case of change of entropy; that is,

$$
\left(\frac{d \rho}{d t}\right)_{m}=\left(\frac{\partial \rho}{\partial s}\right)_{p} \frac{\partial s}{\partial t}=-\left(\frac{\partial \rho}{\partial s}\right)_{p}(\vec{V} \nabla) s .
$$

Here the adiabatic equation (3) is used. For homogeneous (isentropic) medium $(\vec{V} \nabla) s=0$ and from (6) it is seen that change of density of liquid particle is indeed determined by (2). For inhomogeneous medium we can write

$$
(\vec{V} \nabla) s=\vec{V}\left(\frac{\partial s}{\partial P}\right)_{T} \nabla P .
$$

And then, on basis of (6)-(8), the equation of continuity of mass in inhomogeneous medium will take form of

$$
\frac{d \rho}{d t}=-\rho \nabla \vec{V}-\frac{\vec{V} \nabla P}{C_{p}^{2}} .
$$

Quantity $C_{p}^{2}$ has the dimension of squared velocity and equals

$$
C_{p}^{2}=\left[\left(\frac{\partial \rho}{\partial s}\right)_{p}\left(\frac{\partial s}{\partial P}\right)_{T}\right]^{-1}
$$

Real value of the speed of sound in inhomogeneous medium is determined by combination of adiabatic $C_{s}=(\gamma k T / m)^{1 / 2}$ and isobaric $C_{p}$ speeds of sound [2]:

$$
C=\left(\frac{C_{s}^{2} C_{p}^{2}}{C_{s}^{2}+C_{p}^{2}}\right)^{1 / 2}
$$

Thus, the system of equations of hydrodynamics (gas dynamics) in the gravity field of the Earth has the following form:

$$
\begin{aligned}
\rho\left[\frac{\partial \vec{V}}{\partial t}+(\vec{V} \nabla) \vec{V}\right] & =-\nabla P+\rho \vec{g} \\
\frac{\partial \rho}{\partial t}+(\vec{V} \nabla) \rho & =-\rho \nabla \vec{V}-\frac{\vec{V} \nabla P}{C_{p}^{2}} .
\end{aligned}
$$

\section{The Existing Theory of Gravitational Waves}

The system of (12) gives four equations for five unknowns $V_{x}, V_{y}, V_{z}, P$, and $\rho$, and in order for it to be solvable (closed), the existence of another equation that links the disturbances of density $\rho^{\prime}$ and pressure $P^{\prime}$ at constant entropy is required. Such equation is the equation of state of medium:

$$
\rho^{\prime}=\frac{1}{C^{2}} P^{\prime}
$$


where $C$ is expressed by formula (11). According to the current understanding, the medium is considered to be incompressible if the speed of sound in it is infinite, which following formula (13) means $\rho=\rho_{0}=$ const, and from formula (11) we have $C_{s}=C_{p}=\infty$. Under these conditions, from (9), we have

$$
\nabla \vec{V}=0
$$

which is a condition of incompressibility of the medium. Thus, for incompressible fluid, (13) loses its meaning and we have a system of four equations for four unknowns, which is solvable. That is what accounts for the fact that although condition (14) does not apply to liquids (as will be shown below) the existing theory of surface gravity waves gives satisfactory results.

The linearized Euler equation (1) for oscillations with small amplitudes in the absence of a gravitational field, when the entropy at each point of the liquid has the same value (homogeneous, isentropic medium), takes the form

$$
\frac{d \vec{V}}{d t}=\frac{\partial \vec{V}}{\partial t}=-\frac{1}{\rho_{0}} \nabla P .
$$

Having applied to both sides of (15) the operator rot and considering the fact that $\operatorname{rotgrad} P=0$, we obtain

$$
\frac{d}{d t} \operatorname{rot} \vec{V}=0 \Longrightarrow \operatorname{rot} \vec{V}=\text { const. }
$$

The value $\operatorname{rot} \vec{V}$ is referred to as "vorticity" of speed and from (16) it follows that this value is maintained; that is, if it is zero at the starting point, it will remain the same along the whole current line. From preservation of vorticity of speed $\operatorname{rot} \vec{V}=$ const directly follows preservation of circulation of velocity along any closed loop; that is,

$$
\frac{d}{d t} \Gamma(t)=\frac{d}{d t} \oint_{C} \vec{V} \overrightarrow{d l}=0 \Longrightarrow \oint_{C} \vec{V} \overrightarrow{d l}=\text { const. }
$$

This is Thomson's theorem, which is valid only for the ideal isentropic liquid, free from the influence of the gravitational field. If $\operatorname{rot} \vec{V}=0$ in all points of the fluid, the movement is called potential. In this case, there is always a scalar function $\varphi(\vec{r}, t)$, which is called the potential of velocity and satisfies the following condition:

$$
\nabla \varphi(\vec{r}, t)=\vec{V}(\vec{r}, t)
$$

It is obvious that, for an incompressible fluid, when condition (14) is met, the velocity potential satisfies the Laplace equation:

$$
\Delta \varphi(\vec{r}, t)=0
$$

Thomson's theorem contradicts the Helmholtz theorem, according to which in the gravitational field the circulation of speed is maintained [6] (\$1.2). Helmholtz defines a closed circuit parametrically via a vector $\vec{X}(\sigma, t)$, where $0 \leq \sigma \leq 1$ and $\vec{X}(0, t)=\vec{X}(1, t)$. Thus, the choice of the parameter $\sigma$ means selection of the certain liquid particle on the circuit. In this case, the circulation of speed can be written as

$$
\Gamma(t)=\int_{0}^{1} \vec{V} \frac{\partial \vec{X}}{\partial \sigma} d \sigma
$$

From (20), for change of the circulation of speed, the following expression is obtained (see in more detail [6] $(\$ 1.2))$ :

$$
\begin{aligned}
\frac{d \Gamma}{d t}= & \int_{0}^{1}\left[-\frac{1}{\rho_{0}} \frac{\partial P}{\partial \sigma}-g \frac{\partial z}{\partial \sigma}+\frac{1}{2} \frac{\partial V^{2}}{\partial \sigma}\right] d \sigma \\
= & \frac{1}{\rho_{0}}[P(0)-P(1)]+g[z(0)-z(1)] \\
& +\frac{1}{2}\left[V^{2}(1)-V^{2}(0)\right] .
\end{aligned}
$$

According to the author, expression (21) is zero, since on the basis of the cyclicality of parameter $\sigma$ the following equalities are fair: $P(0)=P(1), z(0)=z(1)$, and $V(0)=V(1)$. Actually, it is not the case, because cyclicality of parameter determines the point on the circuit, rather than the values of the physical quantities at this point. These equations are executed only in case velocity has the same value at all points of liquid and if $g=0$. If $g \neq 0$, the contour point (liquid particle) must move strictly in horizontal direction, because any deviation of fluid particle along the axis $Z$ leads to a change of circulation of velocity and movement is nonpotential. This assertion is also made obvious from the fact that, in the gravitational field of the Earth, $z$ component of velocity should depend on the gravitational acceleration $g$; however in this case it is impossible to meet the condition of potentiality of movement $\operatorname{rot} \vec{V}=0 \Rightarrow \partial V_{x} / \partial z=\partial V_{z} / \partial x$.

Let us now discuss how the problems of the linear surface gravity waves generated at the surface of tangential discontinuity $z=0$ between the two fluids are solved in the existing theory. Let us consider the two-dimensional problem, when all variables are dependent on $x, z$, and $t$. Let us route the axis $X$ along the surface of discontinuity and the axis $Z$ perpendicular thereto. Let the upper fluid with infinite depth move relative to the lower liquid with finite, constant depth $h$ at constant speed $\vec{V}_{0}=V_{0} \vec{e}_{x}$. We write the Euler equation (1) in the following form:

$$
\frac{\partial \vec{V}}{\partial t}+\frac{1}{2} \nabla \vec{V}^{2}=-\frac{1}{\rho_{0}} \nabla P+\vec{g} .
$$

Considering the potentiality of the movement and using (18), (22) takes the following form:

$$
\nabla\left[\frac{\partial \varphi}{\partial t}+\frac{1}{2}(\nabla \varphi)^{2}+\frac{P}{\rho_{0}}\right]=\vec{g}
$$


By integrating $x$ and $z$ components of (23) we find

$$
\begin{aligned}
& \frac{P-P_{0}}{\rho_{0}}=\alpha(z, t)-\frac{\partial \varphi}{\partial t}-\frac{1}{2}(\nabla \varphi)^{2}, \\
& \frac{P-P_{0}}{\rho_{0}}=\beta(t)-g z-\frac{\partial \varphi}{\partial t}-\frac{1}{2}(\nabla \varphi)^{2},
\end{aligned}
$$

where $\alpha(z, t)$ and $\beta(t)$ are the arbitrary functions and $P_{0}$ is the equilibrium pressure. From (24) we find easily that

$$
\alpha(z, t)=\beta(t)-g z
$$

and thus

$$
P(x, z, t)=\rho_{0}\left[\beta(t)-g z-\frac{\partial \varphi}{\partial t}-\frac{1}{2}(\nabla \varphi)^{2}\right]+P_{0} .
$$

If the pressure in any liquid particle on the surface of the tangential discontinuity is changed, such change, however small it may be, will spread along the surface in the form of wave and it will no longer be flat. We define the equation of the perturbed surface as $z=\xi(x, t)$. In the existing theory, function $\xi(x, t)$ is given a priori, which we assume is wrong. It should be determined in the process of solving the problem, which we will show below.

On the perturbed surface and on the bottom of the lower liquid the following boundary conditions should be fulfilled:

$$
\begin{aligned}
P_{2}-P_{1} & =-\alpha \frac{\partial^{2} \xi}{\partial x^{2}}, \\
V_{1 z} & =\frac{\partial \xi}{\partial t}+V_{0} \frac{\partial \xi}{\partial x}, \\
V_{2 z} & =\frac{\partial \xi}{\partial t}, \quad z=\xi, \\
V_{2 z} & =0, \quad z=-h .
\end{aligned}
$$

The first of these conditions is called dynamic condition while the rest of the conditions are kinematic conditions. Here, $\alpha$ is the coefficient of the surface tension and indices 1 and 2 denote quantities in the regions $z>0$ and $z<0$, respectively. We introduce the potentials of speeds $\vec{V}_{1}$ and $\vec{V}_{2}$ in the following form:

$$
\begin{aligned}
& \varphi_{1}(x, z, t)=V_{0} x+A \exp (\gamma z) \exp [i(k x-\omega t)], \\
& \varphi_{2}(x, z, t)=B \exp (\delta z) \exp [i(k x-\omega t)] .
\end{aligned}
$$

Note that such definition of the potential is a priori wrong, because, in a gravitational field, $z$ components of velocities, that is, $\partial \varphi_{1} / \partial z$ and $\partial \varphi_{2} / \partial z$, must clearly depend on the gravitational acceleration $g$, which is not taken into account in (28). However, we follow the conventional method and demanding $\varphi_{1}$ and $\varphi_{2}$ to satisfy the Laplace equation and attenuation of perturbations at $z \rightarrow \infty$, we finally have

$$
\begin{array}{r}
\varphi_{1}(x, z, t)=V_{0} x+A \exp (-k z) \exp [i(k x-\omega t)], \\
z>0, \\
\varphi_{2}(x, z, t) \\
=\left[B_{1} \exp (k z)+B_{2} \exp (-k z)\right] \exp [i(k x-\omega t)], \\
z<0 .
\end{array}
$$

Let us define $\xi(x, t)$ as

$$
z=\xi(x, t)=a \exp [i(k x-\omega t)] .
$$

Taking into account (29), (30), and (31) from kinematic conditions (27) at $z=\xi(x, t) \cong 0$ results in

$$
\begin{aligned}
k A & =i\left(\omega-k V_{0}\right) a, \\
k\left(B_{1}-B_{2}\right) & =-i \omega a .
\end{aligned}
$$

From (32) we find the relation between the coefficients $A, B_{1}$, and $B_{2}$ in the following form:

$$
\omega A=\left(\omega-k V_{0}\right)\left(B_{2}-B_{1}\right) .
$$

Substituting (29), (30), and (31) and the value of $a$ from the second equation (32) to (26) and neglecting terms containing squares and products of the coefficients $A, B_{1}$, and $B_{2}$ due to their smallness, from (27), we obtain the following system of linear algebraic equations for these coefficients:

$$
\begin{aligned}
& i\{- {\left[g \frac{\rho_{2}-\rho_{1}}{V_{0}}+\rho_{1}\left(\omega-k V_{0}\right)+\frac{\alpha k^{2}}{V_{0}}\right] A } \\
&-\left[g \frac{\rho_{2}-\rho_{1}}{V_{0}}-\rho_{2} \omega+\frac{\alpha k^{2}}{V_{0}}\right] B_{1} \\
&\left.+\left[g \frac{\rho_{2}-\rho_{1}}{V_{0}}+\rho_{2} \omega+\frac{\alpha k^{2}}{V_{0}}\right] B_{2}\right\} \exp [i(k x-\omega t)] \\
&=\rho_{2} \beta_{2}(t)-\rho_{1} \beta_{1}(t)-\frac{\rho_{1} V_{0}^{2}}{2}, \\
& \omega A+\left(\omega-k V_{0}\right) B_{1}-\left(\omega-k V_{0}\right) B_{2}=0, \\
& \exp (-k h) B_{1}-\exp (k h) B_{2}=0 .
\end{aligned}
$$

Here two kinematic conditions on the surface $z=\xi \cong 0$ are replaced with one condition (33). From (34) it is clear that in order to obtain the dispersion equation $\omega=\omega(k)$, it is necessary to satisfy the following condition:

$$
\rho_{2} \beta_{2}(t)-\rho_{1} \beta_{1}(t)-\frac{\rho_{1} V_{0}^{2}}{2}=0 .
$$

In monograph [5] (\$62) is given a solution of Kelvin problem when $h=\infty$, where $\beta_{1}(t)=\beta_{2}(t)=0$ and as we see, in 
this case, the problem cannot be solved. Therefore, constant term $\rho_{1} V_{0}^{2} / 2$ is artificially introduced in the right part of the first equation (34). In monograph [19] (\$13.7) $\beta_{2}(t)=0$ while $\beta_{1}(t)=-V_{0}^{2} / 2$ is introduced in the expression for potential (29):

$$
\begin{aligned}
\varphi_{1}(x, z, t)= & V_{0} x-\frac{V_{0}^{2} t}{2} \\
& +A \exp (-k z) \exp [i(k x-\omega t)] .
\end{aligned}
$$

Thereafter condition (35) is fulfilled and the problem can be solved. Equating to zero the determinant of system (34) with consideration of (35), we obtain the dispersion equation in the following form:

$$
\begin{aligned}
{\left[\rho_{2}+\right.} & \left.\rho_{1} \operatorname{th}(k h)\right] \omega^{2}-2 \rho_{1} k V_{0} \operatorname{th}(k h) \omega \\
& +k\left[\rho_{1} k V_{0}^{2}-g\left(\rho_{2}-\rho_{1}\right)-\alpha k^{2}\right] \operatorname{th}(k h)=0,
\end{aligned}
$$

the solution of which is

$$
\omega=\frac{\rho_{1} k V_{0} t h(k h) \pm t h^{1 / 2}(k h)\left\{\left[\rho_{2}+\rho_{1} t h(k h)\right]\left[k g\left(\rho_{2}-\rho_{1}\right)+\alpha k^{3}\right]-\rho_{1} \rho_{2} k^{2} V_{0}^{2}\right\}^{1 / 2}}{\rho_{2}+\rho_{1} t h(k h)} .
$$

From (38) follow all the known dispersion relations for special cases. For example, for the air and infinitely deep water $\left(\rho_{2} \gg \rho_{1}\right)$, when $V_{0}=0$, we have

$$
\omega= \pm\left[k\left(g+\frac{\alpha k^{2}}{\rho_{2}}\right)\right]^{1 / 2} .
$$

The wave, dispersion equation of which is expressed by formula (39), is called a capillary-gravity wave. If $k<$ $\left(\rho_{2} g / \alpha\right)^{1 / 2}$, then, for the phase velocity of the wave $U_{p}=\omega / k$, we get

$$
U_{p}=\left(\frac{g}{k}\right)^{1 / 2}
$$

and the wave is purely gravitational. This condition sets the upper limit of wavelength of the capillary wave. For water $\alpha=73 \times 10^{-3} \mathrm{~N} / \mathrm{m}, \rho_{2}=10^{3} \mathrm{~kg} / \mathrm{m}^{3}$ and thus, if the wavelength $\lambda>1.73 \mathrm{~cm}$, then the wave is purely gravitational. For shallow water $(\lambda \gg h, t h(k h) \cong k h)$ at $V_{0}=0$, from (38), we have

$$
U_{p}=\sqrt{g h} .
$$

It is easy to show that the representation of potential in the form of (29) or (36) contradicts its physical sense. Indeed, given that $V_{0}=x / t$, expression (36) can be written as

$$
\begin{aligned}
\varphi_{1} & (x, z, t) \\
& =\frac{V_{0} x}{2}\left\{1+\frac{2 A}{V_{0} x} \exp (-k z) \exp [i(k x-\omega t)]\right\} .
\end{aligned}
$$

Since the coordinate $x$ is not limited in the problem, at $|x|>2 A / V_{0} \varepsilon^{2}$, where $\varepsilon$ is a small dimensionless quantity, square of which is neglected, the second term in the braces must be rejected. This is one more proof that only stationary motion can be potential. Thus, we have convincingly shown that, while studying wave processes in liquids, the movement cannot be considered as a potential not only when $g \neq 0$, but also when $g=0$.

Let us now consider the consequences of the neglect of the second term in (9) on the example of the internal gravitational waves, to which $\$ 13$ of monograph [5] is dedicated. The authors called wave within incompressible liquid located in Earth's gravitational field the internal wave. In connection with incompressibility they neglect change of density related to change of pressure and assume that change of density can only be isobaric, at the expense of change of entropy under mechanic oscillation of inhomogeneous medium; that is,

$$
\rho^{\prime}=\left(\frac{\partial \rho_{0}}{\partial s_{0}}\right)_{p} s^{\prime}
$$

which is similar to formula (7). Thereafter, they write down linearized equations of motion and conservation of mass in the following form:

$$
\begin{aligned}
& \frac{\partial \vec{V}}{\partial t}=\frac{\vec{g}}{\rho_{0}}\left(\frac{\partial \rho_{0}}{\partial s_{0}}\right)_{p} s^{\prime}-\nabla \frac{P^{\prime}}{\rho_{0}}, \\
& \nabla \vec{V}=0 .
\end{aligned}
$$

Having presented all perturbed values of variable values in the form $f^{\prime}(\vec{r}, t)=$ const $\times \exp [i(\vec{k} \vec{r}-\omega t)]$, from (3), (44), and (45), they obtain

$$
\begin{aligned}
i \omega s^{\prime} & =-\vec{V} \nabla s_{0}, \\
-i \omega \vec{V} & =\frac{1}{\rho_{0}}\left(\frac{\partial \rho_{0}}{\partial s_{0}}\right)_{p} s^{\prime} \vec{g}-\frac{i \vec{k}}{\rho_{0}} P^{\prime}, \\
\vec{k} \vec{V} & =0 .
\end{aligned}
$$

Multiplying (47) by vector $\vec{k}$ they receive

$$
i k^{2} P^{\prime}=\left(\frac{\partial \rho_{0}}{\partial s_{0}}\right)_{p} s^{\prime}(\vec{g} \vec{k})
$$

From these equations dispersive equation can easily be obtained in the following form:

$$
\omega^{2}=\omega_{0}^{2} \sin ^{2} \theta
$$


where

$$
\omega_{0}^{2}=-\frac{g}{\rho_{0}}\left(\frac{\partial \rho_{0}}{\partial s_{0}}\right)_{p} \frac{d s_{0}}{d z}
$$

and $\theta$ is angle between vector $\vec{k}$ and axis $Z$. Thus, we get some kind of strange transverse wave, frequency of which depends only on direction of wave vector $\vec{k}$ and it can be of any length. The reason for this paradox is that if, in the equation of mass continuity $d \rho / d t=-\rho \nabla \vec{V}-\vec{V} \nabla P / C_{p}^{2}$, the second summand which related to heterogeneity of medium is not taken into consideration, then the condition $\nabla \vec{V}=0$ means $\rho^{\prime}=0$ or following (43) $s^{\prime}=0$ and then all equations from which dispersive equation (50) is obtained are nulling. If we consider the second summand and ignore the first one, considering liquid to be incompressible, as done in monograph [5], we come to a clear contradiction. Indeed, expressed from (46) $s^{\prime}$ and substituting in (47), we obtain

$$
\vec{V}=\frac{\omega P^{\prime} \vec{k}}{\rho_{0} \omega^{2}-\left(\partial \rho_{0} / \partial s_{0}\right)_{P}\left(\vec{g} \nabla s_{0}\right)},
$$

from which it follows that $\vec{V} \uparrow \uparrow \vec{k}$ that is contrary to condition (48). Therefore, application of the condition of incompressibility $\nabla \vec{V}=0$ for fluids is impossible. In addi tion, as it follows from (44), in this case $\left(\rho^{\prime}=0 \Rightarrow s^{\prime}=\right.$ 0 ), gravitational acceleration disappears from the Euler equation and talk about gravitational waves is impossible. Nevertheless, in the dispersion equations of surface gravity waves, in which condition of potentiality of movement is not used, this acceleration is present that is a consequence of the incorrectness allowed by the authors (see, e.g., [5], \$12). They assume that $\partial V_{z} / \partial t=0$ and solve the system of equations as follows:

$$
\begin{aligned}
\frac{\partial V_{x}}{\partial t} & =-\frac{1}{\rho_{0}} \frac{\partial P}{\partial x}, \\
\frac{1}{\rho_{0}} \frac{\partial P}{\partial z} & =-g, \\
\frac{\partial V_{x}}{\partial x}+\frac{\partial V_{z}}{\partial z} & =0
\end{aligned}
$$

with the boundary condition at $\alpha=0$.

$$
\left.P\right|_{z=\xi}=P_{0} .
$$

Then, by integrating the second equation of (53) in the range $[z ; \xi]$, they find the pressure in form of

$$
P=P_{0}+\rho_{0} g(\xi-z) .
$$

Obviously, this method of solving the problem is erroneous for the following reasons.

Firstly, from condition (54) it follows that pressure is constant on the disturbed liquid surface and thus it is unclear how the wave is propagated in this case.
Secondly, the second equation of system (53) can be written as

$$
\frac{1}{\rho_{0}} \frac{\partial\left(P_{0}+P^{\prime}\right)}{\partial z}=-g
$$

after which, using equilibrium condition of liquid $\partial P_{0} / \partial z=$ $-\rho_{0} g$, we obtain

$$
\frac{\partial P^{\prime}}{\partial z}=0 \Longrightarrow P^{\prime}=\text { const }
$$

that is, if condition (56) is fulfilled, vibration is impossible.

Thirdly, assumption $\partial V_{z} / \partial t=0$ is equivalent to assuming $V_{z}=0$ and consequently $\xi=0$.

These examples conclusively prove that the theory of surface gravity waves is flawed and needs revision.

\section{The Correct Theory of Linear Surface Waves}

Compressibility or incompressibility of the medium is determined by the relation between $C_{s}$ and $C_{p}$. From (11), it follows that if $C_{p}>C_{s}$, then $C \cong C_{s}$ and the medium is compressible; that is, the second term in (9) can be neglected. If $C_{s}>C_{p}$, then $C \cong C_{p}$ and the medium is incompressible; that is, we neglect the first term, assuming that $\nabla \vec{V}=0$. From the definition of isobaric speed of sound we easily find

$$
C_{p}=\left|\rho_{0}\left(\frac{\partial \rho_{0}}{\partial T}\right)_{p}^{-1}\right|\left(\frac{c_{p}}{T}\right)^{1 / 2}=\frac{1}{\beta}\left(\frac{c_{p}}{T}\right)^{1 / 2},
$$

where $\beta=(1 / V)(\partial V / \partial T)_{p}$ is coefficient of thermal expansion and $c_{p}$ is the heat capacity per unit mass of substance at constant pressure. For water, $\beta=1.5 \times 10^{-4} \mathrm{~K}^{-1}$ and $c_{p}=4.19 \times 10^{3} \mathrm{~J} / \mathrm{kg}$, and then from formula (58) we obtain $C_{p}=25210 \mathrm{~m} / \mathrm{sec}$. On the other hand, according to the experiment, the speed of sound in water at the same temperature to a high accuracy is $C=1480 \mathrm{~m} / \mathrm{s}$ and from formula (11) we have $C_{s}=C C_{p} / \sqrt{C_{p}^{2}-C^{2}}=1482.6 \mathrm{~m} / \mathrm{sec}$. As we can see, in water, the speed of sound is almost equal to the adiabatic sound speed and therefore, water is a homogeneous or a compressible medium, for which $\nabla \vec{V} \neq$ 0 . Therefore, for water, as well as for air at sea level, the second term in (9) can be neglected [2]. Let us present all the variables in the form of the sum of fixed and perturbed values as follows:

$$
\begin{gathered}
\vec{V}(x, z, t)=\vec{V}_{0}+\vec{V}^{\prime}(x, z, t), \\
P(x, z, t)=P_{0}+P^{\prime}(x, z, t), \\
\rho(x, z, t)=\rho_{0}+\rho^{\prime}(x, z, t),
\end{gathered}
$$

and then, by linearizing system (12), we obtain

$$
\begin{aligned}
\rho_{0}\left[\frac{\partial \vec{V}}{\partial t}+\left(\vec{V}_{0} \nabla\right) \vec{V}\right] & =-\nabla P+\frac{\vec{g}}{C^{2}} P, \\
\frac{1}{C^{2}}\left[\frac{\partial P}{\partial t}+\left(\vec{V}_{0} \nabla\right) P\right] & =-\rho_{0} \nabla \vec{V} .
\end{aligned}
$$


Here the equation of equilibrium $\nabla P_{0}=\rho_{0} \vec{g}$ and the equation of state $\rho^{\prime}=\left(1 / C^{2}\right) P^{\prime}$ are used while the marks of disturbed values are omitted. Having applied the operator $\nabla$ to the first equation of system (60) and the operator $\partial / \partial t$ to the second equation,

$$
\begin{aligned}
\rho_{0} \frac{\partial}{\partial t} \nabla \vec{V}+\Delta P-\frac{\vec{g}}{C^{2}} \nabla P & =-\rho_{0} \nabla\left[\left(\vec{V}_{0} \nabla\right) \vec{V}\right], \\
\frac{1}{C^{2}} \frac{\partial^{2} P}{\partial t^{2}}+\frac{\vec{V}_{0}}{C^{2}} \frac{\partial}{\partial t} \nabla P & =-\rho_{0} \frac{\partial}{\partial t} \nabla \vec{V},
\end{aligned}
$$

and having determined $\rho_{0} \partial \nabla \vec{V} / \partial t$ from the first equation (61) and substituting in the second, we obtain a generalized equation of gravitational waves in the form of

$$
\begin{aligned}
\Delta P & -\frac{\vec{g}}{C^{2}} \nabla P-\frac{1}{C^{2}} \frac{\partial^{2} P}{\partial t^{2}} \\
& =\frac{\vec{V}_{0}}{C^{2}} \frac{\partial}{\partial t} \nabla P-\rho_{0} \nabla\left[\left(\vec{V}_{0} \nabla P\right) \vec{V}\right],
\end{aligned}
$$

from which the equation for any wave in Earth's gravitational field, including sound wave equation, can be obtained [4]. We will seek for all perturbed quantities in the form $f(x, z, t)=$ $f_{a}(z) \exp [i(k x-\omega t)]$ and take into account that $\vec{V}_{0}=V_{0} \vec{e}_{x}$ and $\nabla\left[\left(\vec{V}_{0} \nabla\right) \vec{V}\right]=V_{0}\left(\partial^{2} V_{x} / \partial x^{2}+\partial^{2} V_{z} / \partial x \partial z\right)$, and then (62) will give the following equation:

$$
\begin{aligned}
& \frac{d^{2} P_{a}(z)}{d z^{2}}+\frac{g}{C^{2}} \frac{d P_{a}(z)}{d z}+\left(\frac{\omega^{2}}{C^{2}}-k^{2}\right) P_{a}(z) \\
& =k V_{0}\left[\rho_{0}\left(k V_{x a}(z)-i \frac{d V_{z a}(z)}{d z}\right)+\frac{\omega}{C^{2}} P_{a}(z)\right] .
\end{aligned}
$$

From the first equation of (60) for $V_{x a}(z)$ and $V_{z a}(z)$ we obtain

$$
\begin{aligned}
V_{x a}(z) & =\frac{k P_{a}(z)}{\rho_{0}\left(\omega-k V_{0}\right)}, \\
V_{z a}(z) & =-i \frac{d P_{a}(z) / d z+g P_{a}(z) / C^{2}}{\rho_{0}\left(\omega-k V_{0}\right)} .
\end{aligned}
$$

Substituting (64) to (63) we will find the equation for the amplitude of perturbed pressure:

$$
\begin{aligned}
& \frac{d^{2} P_{a}(z)}{d z^{2}}+\frac{g}{C^{2}} \frac{d P_{a}(z)}{d z}+\frac{\left(\omega-k V_{0}\right)^{2}-k^{2} C^{2}}{C^{2}} P_{a}(z) \\
& \quad=0
\end{aligned}
$$

At $P_{a}(z)=$ const and $V_{0}=0$, from (65), it follows that the dispersion equation for the sound wave $\omega / k=C$.

We will seek for a solution of (65) in the area $z>0$ in the form $P_{a 1}(z)=A \exp (\gamma k z)$. Then for $\gamma$ we will find

$$
\gamma=-\frac{1}{\theta_{1}}\left[1+\sqrt{1+\theta_{1}^{2}\left(1-\frac{\left(U_{p}-V_{0}\right)^{2}}{C_{1}^{2}}\right)}\right]<0,
$$

where $\theta_{1}=2 k C_{1}^{2} / g$. In the area $z<0$, we will similarly find $P_{a 2}(z)=B_{1} \exp \left(\delta_{1} k z\right)+B_{2} \exp \left(\delta_{2} k z\right)$, where

$$
\begin{aligned}
& \delta_{1}=-\frac{1}{\theta_{2}}\left[1-\sqrt{1+\theta_{2}^{2}\left(1-\frac{U_{p}^{2}}{C_{2}^{2}}\right)}\right]>0, \\
& \delta_{2}=-\frac{1}{\theta_{2}}\left[1+\sqrt{1+\theta_{2}^{2}\left(1-\frac{U_{p}^{2}}{C_{s 2}^{2}}\right)}\right]<0
\end{aligned}
$$

and $\theta_{2}=2 k C_{2}^{2} / g$. Let us consider the condition $\theta_{1}>1$. Given that in the air at sea level $C_{1} \cong 340 \mathrm{~m} / \mathrm{s}$ and $g \approx$ $10 \mathrm{~m} / \mathrm{s}^{2}$, we find $k>8.64 \times 10^{-5} \mathrm{~m}^{-1}$ or $\lambda<0.72 \times 10^{5} \mathrm{~m}$. Thus, this condition embraces the entire range of wavelengths from capillary to tsunami, and the more $k$ is, the better it is performed. For example, if a value of the length of gravitational wave is $\lambda \approx 100 \mathrm{~m}$, for air, $\theta_{1} \approx 0.71 \times 10^{3} \gg 1$ and for water, in which $C_{2} \cong 1500 \mathrm{~m} / \mathrm{s}, \theta_{2} \approx 0.14 \times 10^{5} \gg 1$. It is also evident that $U_{p}^{2} / C_{2}^{2} \ll\left(U_{p}-V_{0}\right)^{2} / C_{1}^{2} \ll 1$ and it is then easy to verify that $\delta_{1}=1$ and $\delta_{2}=\gamma=-1$. Thus, for perturbed pressure, we will, respectively, have the following:

$$
\begin{aligned}
& P_{1}(x, z, t)=A \exp (-k z) \exp [i(k x-\omega t)], \\
& P_{2}(x, z, t) \\
& \quad=\left[B_{1} \exp (k z)+B_{2} \exp (-k z)\right] \exp [i(k x-\omega t)] .
\end{aligned}
$$

For the components of the velocities $\vec{V}_{1}$ and $\vec{V}_{2}$ from formulas (64), neglecting the value $g P_{a} / C^{2}$, we will find

$$
\begin{aligned}
& V_{1 x}(x, z, t)=\frac{k A \exp (-k z)}{\rho_{01}\left(\omega-k V_{0}\right)} \exp [i(k x-\omega t)], \\
& V_{1 z}(x, z, t)=i \frac{k A \exp (-k z)}{\rho_{01}\left(\omega-k V_{0}\right)} \exp [i(k x-\omega t)], \\
& V_{2 x}(x, z, t)=\frac{k\left[B_{1} \exp (k z)+B_{2} \exp (-k z)\right]}{\rho_{02} \omega} \\
& \cdot \exp [i(k x-\omega t)], \\
& V_{2 z}(x, z, t)=-i \frac{k\left[B_{1} \exp (k z)-B_{2} \exp (-k z)\right]}{\rho_{02} \omega} \\
& \cdot \exp [i(k x-\omega t)] .
\end{aligned}
$$

The kinematic conditions on the surface of discontinuity $z=$ $\xi(x, t) \cong 0$ give

$$
\begin{aligned}
\frac{i k A}{\rho_{01}\left(\omega-k V_{0}\right)} \exp [i(k x-\omega t)] & =\frac{\partial \xi}{\partial t}+V_{0} \frac{\partial \xi}{\partial x}, \\
-i \frac{k\left(B_{1}-B_{2}\right)}{\rho_{02} \omega} \exp [i(k x-\omega t)] & =\frac{\partial \xi}{\partial t} .
\end{aligned}
$$


From (70) for $\xi$ we will have

$$
\begin{aligned}
\xi(x, t)= & \frac{1}{V_{0}}\left[\frac{A}{\rho_{01}\left(\omega-k V_{0}\right)}+\frac{B_{1}-B_{3}}{\rho_{02} \omega}\right] \\
& \cdot\{\exp [i(k x-\omega t)]-1\} .
\end{aligned}
$$

Thus, we have defined the function $\xi(x, t)$ from the kinematic conditions on the fracture surface. We see that the amplitude of the surface perturbation depends on the parameters of the task, which is quite natural. Now, using boundary conditions (27), we easily obtain three homogeneous linear equations for coefficients $A, B_{1}$, and $B_{2}$.

$$
\begin{aligned}
& {\left[\frac{\alpha k^{2}}{V_{0} \rho_{01}\left(\omega-k V_{0}\right)}+1\right] A+\left(\frac{\alpha k^{2}}{V_{0} \rho_{02} \omega}-1\right) B_{1}} \\
& -\left(\frac{\alpha k^{2}}{V_{0} \rho_{02} \omega}+1\right) B_{2}=0, \\
& \frac{A}{\rho_{01}\left(\omega-k V_{0}\right)^{2}}+\frac{\omega-k V_{0}}{\rho_{02} \omega^{2}} B_{1}-\frac{\omega-k V_{0}}{\rho_{02} \omega^{2}} B_{2}=0, \\
& \exp (-k h) B_{1}-\exp (k h) B_{2}=0 .
\end{aligned}
$$

Equating the determinant of this system to zero, we will find the dispersion equation in the form of

$$
\left[\rho_{01}\left(\omega-k V_{0}\right)^{2}-\alpha k^{3}\right] t h(k h)+\rho_{02} \omega^{2}=0 .
$$

Despite the fact that the original system of (60) contains acceleration of gravitation $g$, in (73), it is absent. This fact is the specificity of the linear theory, according to which the influence of this parameter is so small that it can be ignored. Equation (73) has no solutions for $\alpha=0$, which means that the linear theory describes only the capillary waves. The solution of this equation at $\rho_{01} \ll \rho_{02}$ is

$\omega$

$$
=k \frac{\rho_{01} V_{0} t h(k h) \pm\left\{t h(k h)\left[\rho_{02} \alpha k-\rho_{01} \rho_{02} V_{0}^{2}\right]\right\}^{1 / 2}}{\rho_{02}},
$$

and therefore, the stability condition of capillary waves is

$$
V_{0} \leq \sqrt{\frac{\alpha k}{\rho_{01}}}
$$

From (75), it is easy to calculate that the wind with the speed of $V_{0}=5 \mathrm{~m} / \mathrm{s}$ will blow off capillary waves whose length $\lambda>$ $1.6 \mathrm{~cm}$. At $V_{0}=0$, from (74), we have

$$
U_{p}=\frac{\omega}{k}= \pm \sqrt{\frac{k \alpha}{\rho_{02}} t h(k h)} .
$$

Solution (76) refutes the existing opinion that the capillary waves are generated only in the deep water. We see that they are generated in deep $(k h>1, t h(k h) \approx 1)$ as well as in shallow $(k h<1, t h(k h) \approx k h)$ water. In the first case the dispersion equation has the following form:

$$
\omega= \pm k \sqrt{\frac{k \alpha}{\rho}}
$$

and in the second, it has the following form:

$$
\omega= \pm k^{2} \sqrt{\frac{\alpha h}{\rho}} .
$$

Since now there are no conditions limiting the length of the capillary waves, let us consider the perturbation with wavelength $\lambda=0.1 \mathrm{~m}\left(k=62.8 \mathrm{~m}^{-1}\right)$, for which, from formulas (77) and (78), we find that in deep water $(h \geq 0.5 \mathrm{~m})$ $\omega=0.13 \mathrm{~s}^{-1}$ and $U_{p}=2 \mathrm{~cm} / \mathrm{sec}$ and in shallow water $(h=$ $0.05 \mathrm{~m}) \omega=0.07 \mathrm{sec}^{-1}$ and $U_{p}=1 \mathrm{~cm} / \mathrm{sec}$. As we can see, as the depth reduces 10 times, the frequency and phase velocity of capillary wave fall 2 times.

\section{Conclusion}

It was noted above that the existing theory of gravitational waves, which describes the propagation of mechanical vibrations in the medium under the influence of Earth's gravitational field, gives satisfactory results. By saying so we meant that they can be explained in terms of physics, but this does not mean that they are correct. For example, the condition $k<\left(\rho_{2} g / \alpha\right)^{1 / 2}$ according to which the capillary is only the waves whose length does not exceed $1.73 \mathrm{~cm}$ is not confirmed by experiment. There is a classic experiment in which a steel needle does not sink in a glass, filled to the brim with water. This is because although the diameter of glass greatly exceeds the above specified length, the force of surface tension acts, which balances the pressure produced by the needle. Expression (41) for the phase velocity of gravitational waves in shallow water $U_{p} \sqrt{g h}$ which is obtained from incorrect linear theory and is used to determine the speed of tsunami waves (see, e.g., [20]) also seems doubtful. This formula gives satisfactory results in the distance from the shore, but it shows that the speed of wave of tsunami should tend to zero at run-up onto the shore, while it has enormous destructive energy. We have shown that, in the linear theory, phase velocity of surface wave is independent of the gravitational acceleration, and therefore, the speed of tsunami waves must be determined from the nonlinear theory. Since this wave propagates at the interface between water and air, its speed must be dependent on the thermodynamic parameters of these two media and should accelerate while running up to the shore.

From the foregoing it follows that our calculations and arguments leave no doubt that they should be taken into account in the study of dynamic processes in liquids and gases. 


\section{Competing Interests}

The author declares no competing interests.

\section{References}

[1] V. G. Kirtskhalia, "On gravity waves on the surface of tangential discontinuity," Applied Physics Research, vol. 6, no. 2, pp. 109-117, 2014.

[2] V. G. Kirtskhalia, "Speed of sound in atmosphere of the Earth," Open Journal of Acoustics, vol. 2, no. 2, pp. 80-85, 2012.

[3] V. G. Kirtskhalia, "The new determination of the criteria of compressibility and incompressibility of medium," Journal of Modern Physics, vol. 4, no. 8, pp. 1075-1079, 2013.

[4] V. Kirtskhalia, "Sound wave as a particular case of the gravitational wave," Open Journal of Acoustics, vol. 2, no. 3, pp. 115-120, 2012.

[5] L. D. Landau and E. N. Lifshitz, Theoretical Physics, vol. 6 of Hydrodynamics, Nauka, Moscow, Russia, 1988.

[6] J. J. Stoker, Water Waves, Interscience, New York, NY, USA, 1957.

[7] J. M. Hassan, T. A. Mohamed, W. S. Mohammed, and W. H. Alawee, "Modeling the uniformity of manifold with various configurations," Journal of Fluids, vol. 2014, Article ID 325259, 8 pages, 2014.

[8] R. Chand and G. C. Rana, "Double diffusive convection in a layer of Maxwell viscoelastic fluid in porous medium in the presence of Soret and Dufour effects," Journal of Fluids, vol. 2014, Article ID 479107, 7 pages, 2014.

[9] M. G. Rabby, S. P. Shupti, and M. M. Molla, "Pulsatile non-newtonian laminar blood flows through arterial double stenoses," Journal of Fluids, vol. 2014, Article ID 757902, 13 pages, 2014.

[10] N. T. M. Eldabe, B. M. Agoor, and H. Alame, "Peristaltic motion of non-Newtonian fluid with heat and mass transfer through a porous medium in channel under uniform magnetic field," Journal of Fluids, vol. 2014, Article ID 525769, 12 pages, 2014.

[11] M. Singh and R. K. Gupta, "Double-diffusive convection in presence of compressible rivlin-ericksen fluid with fine dust," Journal of Fluids, vol. 2014, Article ID 714150, 6 pages, 2014.

[12] G. I. Ogilvie, "Astrophysical fluid dynamics," Journal of Plasma Physics, vol. 82, no. 3, 2016.

[13] O. Troshkin, "On stable plane vortex flows of an ideal fluid," Mathematics and Statistics, vol. 4, no. 2, pp. 47-57, 2016.

[14] M. Campos Pinto and F. Charles, "Adaptive wavelet simulation of weakly compressible flow in a channel with a suddenly expanded section," Proceedings and Surveys, vol. 53, pp. 38-48, 2016.

[15] C. J. Cotter, J. Eldering, D. D. Holm, H. O. Jacobs, and D. M. Meier, "Weak dual pairs and Jetlet methods for ideal incompressible fluid models in $n \geq 2$ dimensions," Journal of Nonlinear Science, vol. 26, no. 6, pp. 1723-1765, 2016.

[16] F. Bouchut, Y. Jobic, R. Natalini, R. Occelli, and V. Pavan, "Second-order entropy satisfying BGK-FVS schemes for incompressible Navier-Stokes equations," HAL Id: hal-01337030, https://hal-upec-upem.archives-ouvertes.fr/hal-01337030.

[17] J. M. Cubos-Ramírez, J. Ramírez-Cruz, M. Salinas-Vázquez, W. Vicente-Rodríguez, E. Martinez-Espinosa, and C. LagarzaCortes, "Efficient two-phase mass-conserving level set method for simulation of incompressible turbulent free surface flows with large density ratio," Computers \& Fluids, vol. 136, pp. 212227, 2016.
[18] K. Rehman and Y. Cho, "Bed evolution under rapidly varying flows by a new method for wave speed estimation," Water, vol. 8, no. 5, article 212, 2016.

[19] G. Whitham, Linear and Nonlinear Waves, John Wiley \& Sons (Wiley-Interscience), New York, NY, USA, 1974.

[20] Z. Kowalik, Introduction to Numerical Modeling of Tsunami Waves, Institute of Marine Science, University of Alaska, Fairbank, Alaska, USA, 2012. 

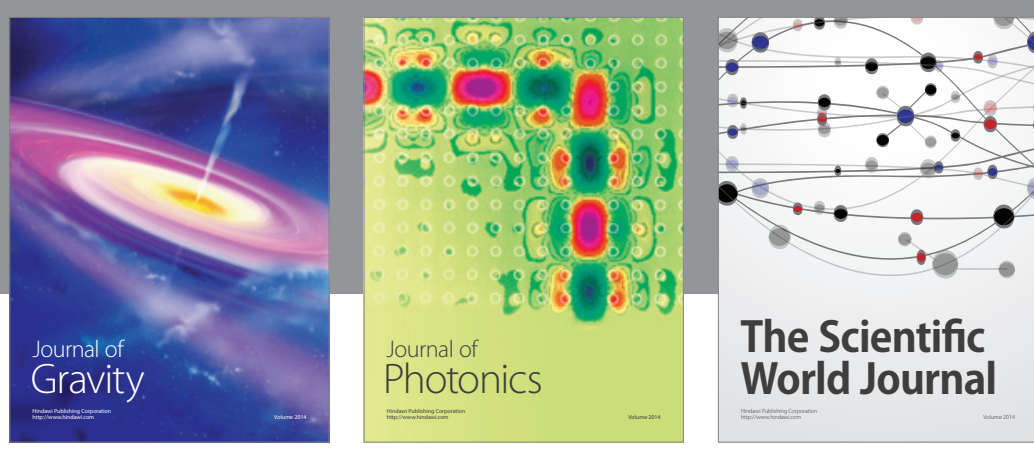

The Scientific World Journal
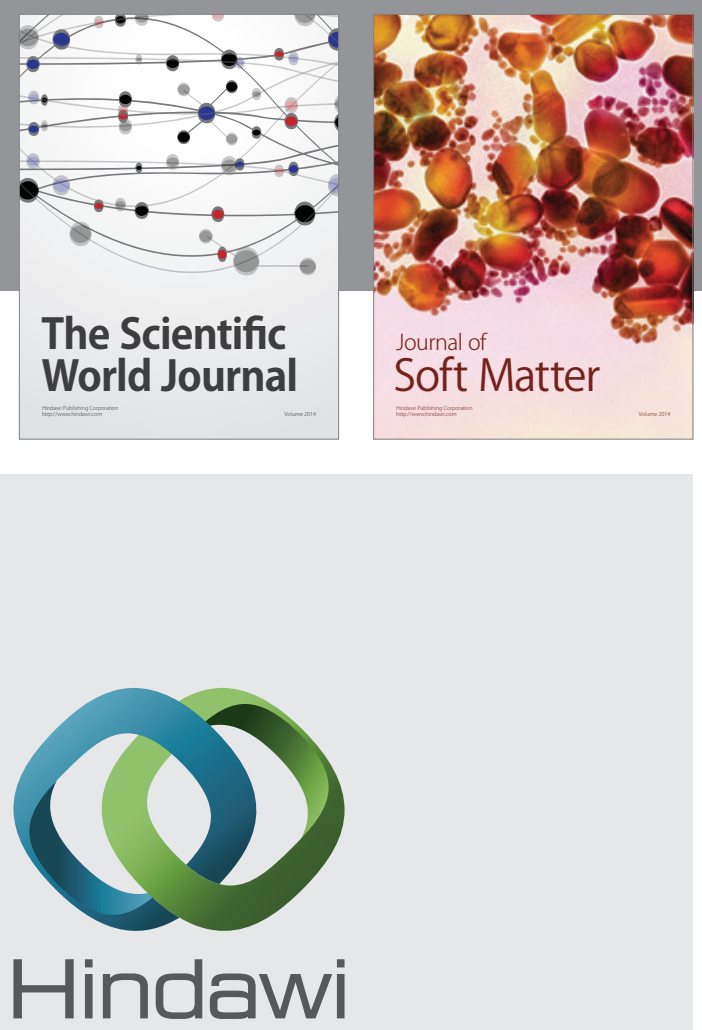

Submit your manuscripts at

http://www.hindawi.com

nternational Journal of

Statistical Mechanics
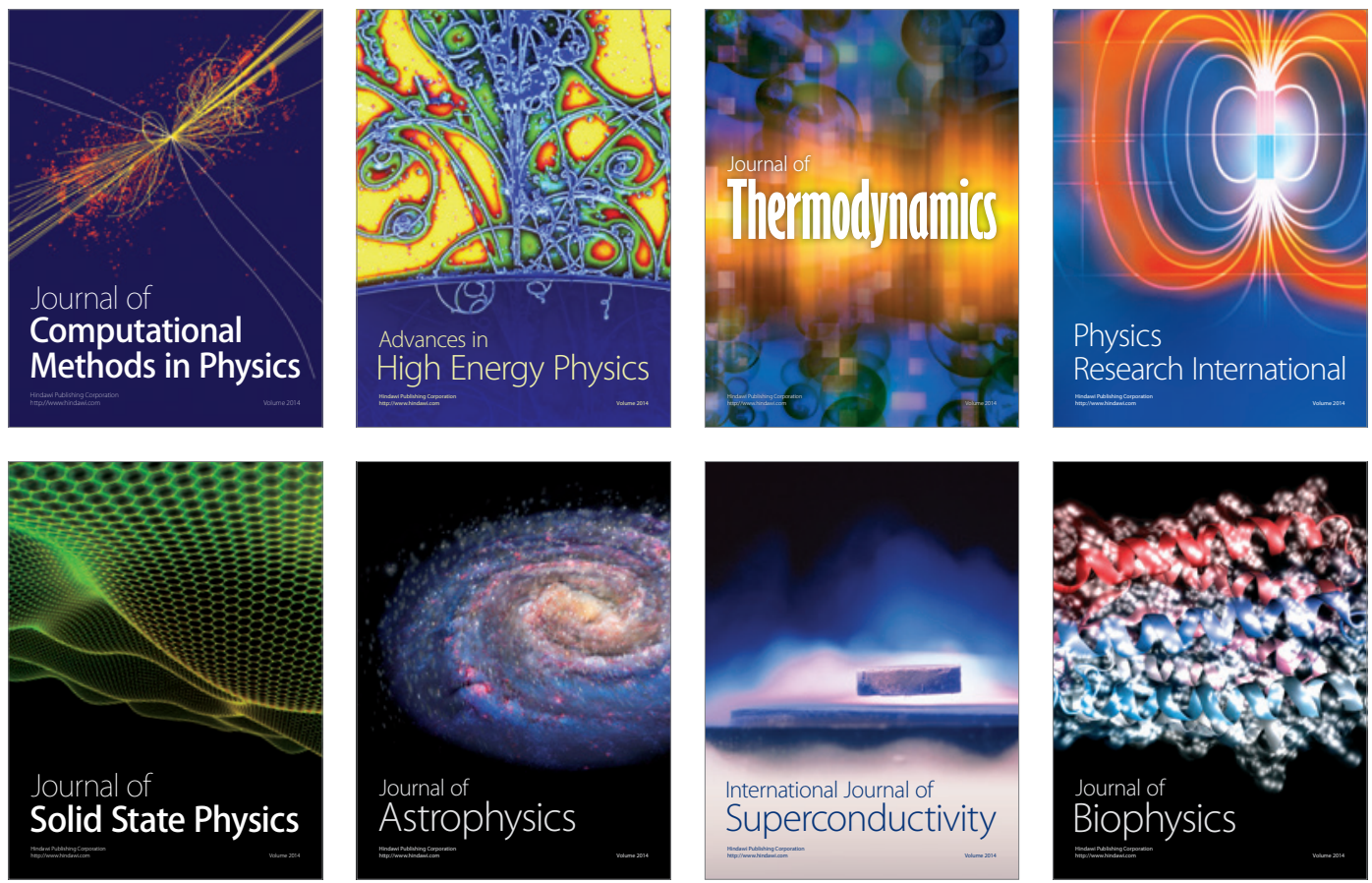
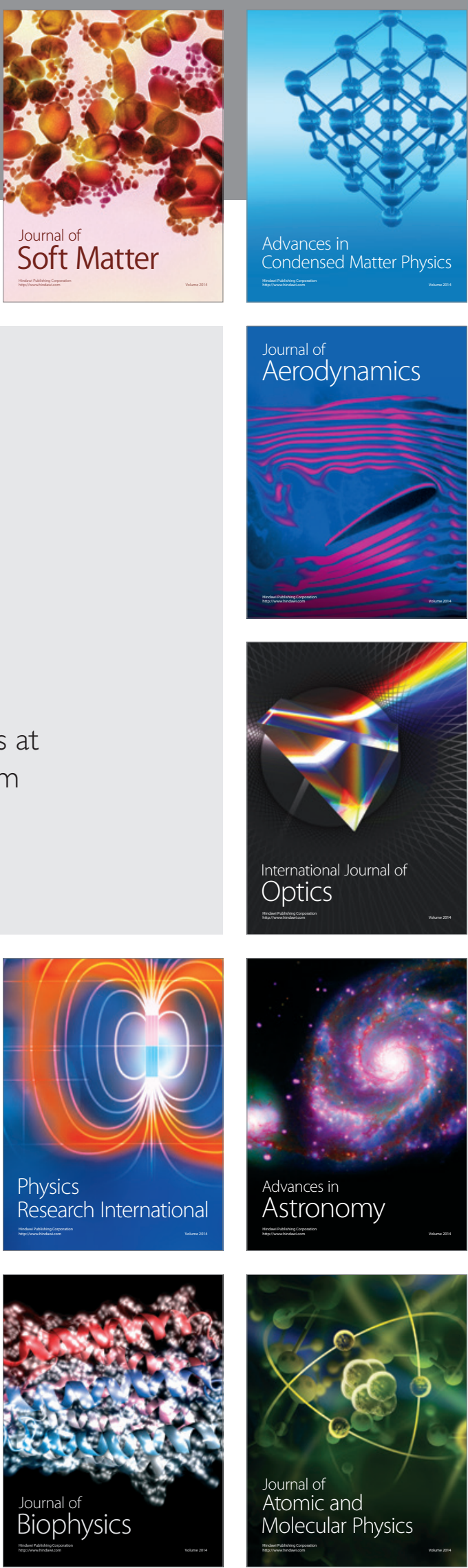